\title{
The role of radioactive iodine therapy in papillary thyroid cancer: an observational study based on SEER
}

\author{
Jianing Tang' \\ Deguang Kong² \\ Qiuxia Cui' \\ Kun Wang ${ }^{3}$ \\ Dan Zhang ${ }^{3}$ \\ Xing Liao' \\ Yan Gong ${ }^{4}$ \\ Gaosong Wu'
}

'Department of Thyroid and Breast Surgery, Zhongnan Hospital of Wuhan University, Wuhan, China; ${ }^{2}$ Department of General Surgery, Zhongnan Hospital of Wuhan University, Wuhan, China; ${ }^{3}$ Department of Breast and Thyroid Surgery, Tongji Hospital of Tongji Medical College, Huazhong University of Science and Technology, Wuhan, China; ${ }^{4}$ Department of Biological Repositories, Zhongnan Hospital of Wuhan University, Wuhan, China

Correspondence: Yan Gong Department of Biological Repositories, Zhongnan Hospital of Wuhan University, 169 Donghu Road, Wuhan, Hubei 43007I, China

Tel +86 I39 7I53 35I2

Email yan.gong@whu.edu.cn

Gaosong Wu

Department of Thyroid and Breast Surgery, Zhongnan Hospital of Wuhan University, 169 Donghu Road, Wuhan, Hubei 43007I, China

Tel +86 I38 7| 444606

Email wugaosongtj@I63.com
This article was published in the following Dove Press journal: OncoTargets and Therapy

Background: Papillary thyroid cancer (PTC) is a common endocrine malignancy with relatively good prognosis. Radioactive iodine (RAI) is considered effective for patients with total or nearly total thyroidectomy, but the beneficial effects of RAI are still controversial.

Materials and methods: To determine whether RAI therapy could improve the survival rates of PTC patients, we conducted a retrospective analysis using data from the National Cancer Institute's Surveillance, Epidemiology, and End Results (SEER) program. Disease-specific survival (DSS) was obtained using multivariate Cox proportional hazard regressions.

Results: DSS was improved by RAI ablation in patients with tumor $>2 \mathrm{~cm}$, age $>45$ years and gross extrathyroidal or lymph node metastasis. In a further analysis, RAI therapy did not improve the DSS in patients with tumor $<2 \mathrm{~cm}$ except those with distant metastasis. For patients with tumor $>2 \mathrm{~cm}$, those involving gross extrathyroidal extension, age $>45$ years or disease in the lymph nodes, DSS was improved after RAI therapy. Patients with distant metastasis always benefited from RAI ablation.

Conclusion: RAI ablation should be recommended to patients with tumor $<2 \mathrm{~cm}$ and distant metastasis or patients with tumor $>2 \mathrm{~cm}$ and one of the following risk factors: gross extrathyroidal extension, age $>45$ years, lymph node and distant metastases.

Keywords: RAI, prognosis, tumor size, metastasis, extension, age

\section{Introduction}

Thyroid cancer is a common endocrine malignancy. Over the past 3 decades, the incidence of thyroid cancer increased by $211 \%$ in the USA, driven mostly by the increase of papillary thyroid cancer (PTC). With the high incidence of thyroid cancer, mortality rate for advanced-stage PTC has also increased.,

It is widely agreed that PTC demonstrates relatively indolent clinical behavior. The improvements in diagnosis and treatment of PTC provide an excellent prognosis, with $90 \%$ survival rate at 10 years. ${ }^{3-5}$ However, it remains a challenge to establish a standard treatment strategy for patients with PTC. To determine whether patients need more aggressive treatment, risk stratification systems were set up to stratify patients into low and high risk of recurrence or death of thyroid cancer. ${ }^{6}$ In the current American Thyroid Association (ATA) Guidelines, radioactive iodine (RAI) is not routinely recommended after thyroidectomy for the low risk patients, but it is considered for ATA intermediate and high risk patients. ${ }^{7}$ For patients with low risk factors, total thyroidectomy can remove the remnant, thus postoperative RAI ablation has no further effect on prognosis improvement. ${ }^{8}$ It was reported that RAI only affected the survival of patients $>45$ years old with primary tumors $>2 \mathrm{~cm}$ and lymph node and 
distant metastases. ${ }^{9}$ Surgical resection followed by RAI is often advised for high risk tumors. While Mazzaferri and Jhiang demonstrated that RAI therapy significantly reduced recurrence rate and cancer-related death in patients with tumors $>1.5 \mathrm{~cm},{ }^{10}$ Jonklaas et al stated that RAI therapy benefited all patients except those with stage 1 disease. ${ }^{11}$ No statistic differences of recurrence and death rates were reported between the ${ }^{131} \mathrm{I}$ group and the no- ${ }^{131} \mathrm{I}$ group in a study recruiting 1,542 patients from Mayo Clinic. ${ }^{12}$ RAI did not affect the survival rate in patients with small intrathyroid tumors. Therefore, RAI therapy was only recommended for patients with residual disease. ${ }^{13,14}$ In most countries, postsurgical RAI ablation is recommended for PTC patients with tumors $>1 \mathrm{~cm}$. Due to the lack of long-term randomized trials and inconsistence of previous studies, the beneficial effects of RAI are still controversial. ${ }^{15}$

To further investigate the effects of RAI therapy on patients with PTC, we conducted a retrospective analysis using data from the National Cancer Institute's Surveillance, Epidemiology, and End Results (SEER) Program. The objective of this study was to determine whether RAI therapy could improve the long-term survival rates. Furthermore, we aimed to determine whether a cohort of patients with specific risk factors could be identified, and whether RAI therapy was associated with an improved survival rates for those patients.

\section{Materials and methods}

A retrospective cohort analysis using the SEER database from the National Cancer Institute was performed. Patients were selected between 2003 and 2013. Because of the different clinical behaviors between classical type and follicular variant of PTC, histologic subtypes of PTC were limited using the International Classification of Diseases for Oncology, 3rd edition $^{32}$ (ICD-O-3) as follows: 8050/3: papillary carcinoma, not otherwise specified (NOS); 8260/3: papillary adenocarcinoma, NOS; 8343/3: papillary carcinoma, encapsulated.

Patients whose information of tumor size, extension, lymph node and distant metastases was blank or unknown were excluded from this study. Cases with or without RAI ablation after total or nearly total thyroidectomy were eligible for further analysis.

Demographic data included sex (male and female), age at diagnosis ( $<45$ or $\geq 45$ years), race (white, black, Asian, other or unknown) and region (East, Northern Plains, Pacific Coast, Southwest or Alaska). Tumor size cutoffs from 1 to $4 \mathrm{~cm}$ were examined in $1 \mathrm{~cm}$ intervals to determine whether a tumor size threshold could be identified above which RAI therapy was beneficial to survival rates. The cancer characteristic of tumor extension was categorized as intrathyroidal (codes 100, 200, 300, 400), minimal extrathyroidal (code 450) and gross extrathyroidal (codes 480, 500, 520, 550, 600, 620, 650, 700, $720,730,800)$. Lymph node metastasis was classified into 2 groups: negative (code 000) and positive (codes 120, 135, $140,155,158,160)$. Distant metastasis included no distant metastasis diseases (code 00) and distant metastasis diseases (codes 12, 40, 51, 60). Treatment characteristics included surgery (code 40: subtotal or nearly total thyroidectomy, code 50: total thyroidectomy) and radiation therapy (none or radioactive iodine therapy). All the variables were defined using the SEER specific codes.

To determine the tumor size threshold and assess the association between tumor size and RAI therapy, the analysis was firstly stratified by tumor size (by $1 \mathrm{~cm}$ intervals). We further stratified the patients based on tumor extension, age, lymph node and distant metastases to investigate the effects of RAI on patients with specific features.

Patient demographics, cancer- and treatment-related characteristics were compared between no RAI and RAI therapy groups using chi-square or Fisher's exact tests. In our study, the overall survival (OS) and disease-specific survival (DSS) were obtained using multivariate Cox proportional hazard regressions. A Cox proportion hazards model was used to assess the association between all the variables and survival. Stratified analyses were performed and Cox proportion hazards model was obtained to investigate the effects of RAI therapy. Adjusted hazard ratios (aHR) with 95\% confidence intervals were generated to quantify the strength of the relative risk, and aHR $>1.0$ represented a worse prognosis than those $<1.0$. All the tests and performed were 2-tailed, and $P<0.05$ was considered statistically significant. All statistical analyses were performed using SPSS 19.0 (IBM Corporation, Armonk, NY, USA).

\section{Ethics statement}

This was a retrospective cohort analysis using data from the SEER database which was designed and maintained by the National Cancer Institute. Research was limited to secondary use of information previously collected in the course of normal care and data were anonymized before the conduction of statistical analyses. This article does not contain any studies with human participants or animals performed by any of the authors.

\section{Results}

\section{Patient characteristics}

A total of 41,287 cases were eligible for our study. The median follow-up time was 47.2 months. Among the total 
death rate of $1,423(3.4 \%)$, only 261 patients died from thyroid cancer $(0.6 \%)$. Characteristics of patients are compared between the treatment subgroups in Table 1. Over half of the patients underwent postoperative RAI therapy. Patients with larger tumors, extrathyroidal extension, lymph node or distant metastases were more likely to be given RAI ablation after total or nearly total thyroidectomy.

\section{Survival analysis}

The aHRs for OS and DSS are listed in Table 2 to investigate the clinical significances. The multivariate models

Table I Patient characteristics within subgroups

\begin{tabular}{|c|c|c|c|}
\hline Variables & $\begin{array}{l}\text { No RAI therapy } \\
N=18,222(\%)\end{array}$ & $\begin{array}{l}\text { RAl therapy } \\
\mathrm{N}=23,065 \text { (\%) }\end{array}$ & P-value* \\
\hline $\begin{array}{l}\text { Median follow-up } \\
\text { (months) }\end{array}$ & 42.2 & 50.8 & \\
\hline Age at diagnosis, years & & & $<0.001$ \\
\hline$<45$ & $7,078(38.8)$ & $10,534(45.7)$ & \\
\hline$\geq 45$ & $\mathrm{II}, \mid 44(6 \mid .2)$ & $|2,53|(54.3)$ & \\
\hline Sex & & & $<0.001$ \\
\hline Male & 3,596 (19.7) & $5,695(24.7)$ & \\
\hline Female & $14,626(80.3)$ & $17,370(75.3)$ & \\
\hline Race & & & $<0.001$ \\
\hline White & $14,995(82.3)$ & I8,972 (82.3) & \\
\hline Black & $1,116(6.1)$ & $903(3.9)$ & \\
\hline Asian & $1,689(9.3)$ & $2,655(11.5)$ & \\
\hline Other & $275(1.5)$ & $445(1.9)$ & \\
\hline Unknown & $147(0.8)$ & $90(0.4)$ & \\
\hline Region & & & $<0.001$ \\
\hline East & $7,255(39.8)$ & $7,532(32.7)$ & \\
\hline Pacific Coast & $8,293(45.5)$ & $11,839(5 \mid .3)$ & \\
\hline Southwest & $1,169(6.4)$ & $\mathrm{I}, 653(7.2)$ & \\
\hline Northern Plains & $\mathrm{I}, 500(8.2)$ & $2,021(8.8)$ & \\
\hline Alaska & $5(0.0)$ & $20(0.1)$ & \\
\hline Surgery & & & $<0.001$ \\
\hline $\begin{array}{l}\text { Subtotal or near total } \\
\text { thyroidectomy }\end{array}$ & 991 (5.4) & $642(2.8)$ & \\
\hline Total thyroidectomy & $|7,23|(94.6)$ & $22,423(97.2)$ & \\
\hline Tumor size $(\mathrm{cm})$ & & & $<0.001$ \\
\hline$\leq 2$ & I4,8|7 (8I.3) & $14,629(63.4)$ & \\
\hline$>2$ & $3,405(18.7)$ & $8,436(36.6)$ & \\
\hline Tumor extension & & & $<0.001$ \\
\hline Intrathyroidal & I6,230 (89.1) & I6,708 (72.4) & \\
\hline Minimal extrathyroidal & $851(4.7)$ & $2,651(11.5)$ & \\
\hline Gross extrathyroidal & $\mathrm{I},|4| \mathrm{I}(6.3)$ & $3,706(16.1)$ & \\
\hline Lymph node metastases & & & $<0.001$ \\
\hline Negative & I5,634 (85.8) & $14,290(62.0)$ & \\
\hline Positive & $2,588(14.2)$ & $8,775(38.0)$ & \\
\hline Distant metastases & & & $<0.001$ \\
\hline None & $18,154(99.6)$ & $22,831(99.0)$ & \\
\hline Yes & $68(0.4)$ & $234(1.0)$ & \\
\hline Status & & & $<0.001$ \\
\hline Alive & $17,520(96.1)$ & 22,344 (96.9) & \\
\hline Dead & $702(3.9)$ & $721(3.1)$ & \\
\hline Thyroid cancer & $94(0.5)$ & $167(0.7)$ & \\
\hline Other & $608(3.3)$ & $554(2.4)$ & \\
\hline
\end{tabular}

Note: *P-values were calculated by Pearson chi-squared or Fisher's exact testing. Abbreviation: $\mathrm{RAl}$, radioactive iodine. demonstrated that older age, larger tumor, extrathyroidal extension, lymph node and distant metastases had negative effects on the survival when controlling for the remaining variables $(P<0.05)$. RAI therapy improved both OS (aHR $=$ $0.586, P<0.001)$ and DSS $(\mathrm{aHR}=0.600, P<0.001)$.

\section{Subgroup analysis of RAI therapy}

We investigated the beneficial effects of RAI on patients with different features. Patients were stratified based on tumor size, age, extension, and metastasis. To determine the tumor size threshold for which RAI therapy could benefit the survival, tumor size was tested by $1 \mathrm{~cm}$ intervals between 1 and $4 \mathrm{~cm}$. For patients with tumors $<2 \mathrm{~cm}$, RAI improved OS but did not benefit DSS. However, for those with tumors $>2 \mathrm{~cm}$, RAI therapy could significantly improve the OS and DSS. Similar results were found when patients were classified by age ( $<45$ or $\geq 45$ years), extension (intrathyroidal, minimal extrathyroidal or gross extrathyroidal), or lymph nodes metastasis (negative or positive). DSS was improved in older age, gross extrathyroidal, and lymph node groups but not the distant metastasis group (Table 3).

To further examine the associations between RAI therapy and DSS, patients were stratified (Figure 1). RAI therapy did not improve the DSS in all tumor $<2 \mathrm{~cm}$ groups, except for patients with distant metastasis. For patients with tumors $>2 \mathrm{~cm}$, DSS benefited from RAI therapy only for those involving gross extrathyroidal extension, age $>45$ years or with disease in the lymph nodes; DSS was improved in tumor $>2 \mathrm{~cm}$ whether or not patients had distant metastasis (Figures 2 and 3).

\section{Discussion}

Although postoperative RAI therapy is widely recommended for differentiated thyroid carcinoma, ${ }^{15}$ the beneficial effects of RAI have been debated. In the current ATA Guidelines, RAI adjuvant therapy was not routinely recommended for low risk patients. It was considered after total thyroidectomy in ATA intermediate risk level differentiated thyroid cancer patients and high risk differentiated thyroid cancer patients. The European Thyroid Association (ETA) guideline advised RAI for patients with T3/T4, N1 or M1 disease, while for young patients ( $<18$ years) and patients with primary tumors between 1 and $2 \mathrm{~cm}$ without metastasis, it only gave a relative indication for RAI therapy. ${ }^{16}$ The Society of Nuclear Medicine (SNM) Procedure Guideline recommended postoperative RAI for patients with tumor $>1.5 \mathrm{~cm}$, or tumor $<1.5 \mathrm{~cm}$ with metastasis. ${ }^{17}$ The European Association of Nuclear Medicine Guidelines considered RAI ablation as a standard procedure in patients with differentiated thyroid 
Table 2 Cox proportional hazards regression model analysis of overall survival (OS) and disease-specific survival (DSS)

\begin{tabular}{|c|c|c|c|c|}
\hline \multirow[t]{2}{*}{ Variables } & \multicolumn{2}{|l|}{ OS } & \multicolumn{2}{|l|}{ DSS } \\
\hline & aHR $(95 \% \mathrm{Cl})$ & $P$-value* & aHR $(95 \% \mathrm{Cl})$ & $P$-value* \\
\hline \multicolumn{5}{|l|}{ Age at diagnosis, years } \\
\hline$<45$ & Reference & & Reference & \\
\hline$\geq 45$ & $6.730(5.649,8.018)$ & $<0.00 \mathrm{I}$ & $20.332(10.774,38.368)$ & $<0.001$ \\
\hline \multicolumn{5}{|l|}{ Sex } \\
\hline Male & Reference & & Reference & \\
\hline Female & $0.567(0.508,0.633)$ & $<0.00 \mathrm{I}$ & $0.656(0.509,0.844)$ & 0.001 \\
\hline \multicolumn{5}{|l|}{ Race } \\
\hline White & Reference & & Reference & \\
\hline Black & $1.237(0.988,1.549)$ & 0.064 & $1.065(0.560,2.026)$ & 0.848 \\
\hline Asian & $0.734(0.599,0.899)$ & 0.003 & $0.844(0.575,1.238)$ & 0.385 \\
\hline Other & $1.231(0.832,1.821)$ & 0.298 & $1.349(0.628,2.901)$ & 0.443 \\
\hline \multicolumn{5}{|l|}{ Region } \\
\hline East & Reference & & Reference & \\
\hline Pacific Coast & $0.930(0.826,1.047)$ & 0.229 & $\mathrm{I} .087(0.8 \mathrm{I} 8, \mathrm{I} .445)$ & 0.566 \\
\hline Southwest & $0.909(0.722,1.143)$ & 0.413 & $0.546(0.279,1.069)$ & 0.077 \\
\hline Northern Plains & I.I $39(0.948,1.368)$ & 0.165 & $1.113(0.697,1.776)$ & 0.654 \\
\hline Alaska & $1.453(0.342,6.173)$ & 0.613 & $0.000(0.000,6.575 \mathrm{E}+266)$ & 0.973 \\
\hline \multicolumn{5}{|l|}{ Surgery } \\
\hline Subtotal or near total thyroidectomy & Reference & & Reference & \\
\hline Total thyroidectomy & $0.815(0.656,1.012)$ & 0.064 & $0.590(0.354,0.986)$ & 0.044 \\
\hline \multicolumn{5}{|l|}{ RAl therapy } \\
\hline None & Reference & & Reference & \\
\hline Yes & $0.586(0.525,0.655)$ & $<0.001$ & $0.600(0.46 \mathrm{I}, 0.780)$ & $<0.00$ I \\
\hline \multicolumn{5}{|l|}{ Tumor size $(\mathrm{cm})$} \\
\hline$\leq 2$ & Reference & & Reference & \\
\hline$>2$ & $\mathrm{I} .493(1.331,1.674)$ & $<0.001$ & $3.704(2.758,4.975)$ & $<0.001$ \\
\hline \multicolumn{5}{|l|}{ Extension } \\
\hline Intrathyroidal & Reference & & Reference & \\
\hline Minimal extrathyroidal & $1.201(0.985,1.463)$ & 0.070 & $2.325(1.517,3.562)$ & $<0.001$ \\
\hline Gross extrathyroidal & $1.995(1.740,2.288)$ & $<0.001$ & $5.047(3.682,6.916)$ & $<0.00$ I \\
\hline \multicolumn{5}{|l|}{ Lymph node metastases } \\
\hline Negative & Reference & & Reference & \\
\hline Positive & $1.290(1.137,1.463)$ & $<0.001$ & $2.103(1.589,2.784)$ & $<0.001$ \\
\hline \multicolumn{5}{|l|}{ Distant metastases } \\
\hline None & Reference & & Reference & \\
\hline Yes & $5.398(4.291,6.790)$ & $<0.001$ & I0.257 (7.422, I4.176) & $<0.001$ \\
\hline
\end{tabular}

Note: $* P$-values calculated by multivariate Cox analysis.

Abbreviations: aHR, adjusted hazard ratio (adjusted for age at diagnosis, sex, race, region, tumor size, extension, lymph nodes metastases, distant metastases, radiotherapy and surgery); RAI, radioactive iodine.

cancer after total or nearly total thyroidectomy, except for patients with unifocal PTC $<1 \mathrm{~cm}$ without metastasis, thyroid capsule invasion, history of radiation exposure or unfavorable histology. ${ }^{8}$ Due to the lack of long-term randomized trails and the use of different stratification systems, it is not easy to find the optimal treatment for patients.

In our study, we conducted a retrospective, populationbased cohort analysis using the SEER database. Patients were stratified into different subgroups based on risk factors. DSS was improved by RAI only in patients with tumors $>2 \mathrm{~cm}$, gross extrathyroidal extension, older age ( $>45$ years) and lymph node metastasis. Distant metastasis did not affect the outcomes of RAI due to the low percentage of patients with distant metastasis $(0.7 \%)$. In patients with tumors $>2 \mathrm{~cm}$, RAI could improve the DSS for those with gross extrathyroidal extension, lymph node metastases, distant metastases or aged $>45$ years. Although patients without distant metastases in this group also benefited from RAI ablation, they should be further analyzed based on their characteristics (age, tumor extension, lymph nodes metastases), because the majority of patients did not have distant metastases. In patients with tumors $<2 \mathrm{~cm}$, DSS was not affected by the use of RAI regardless of age, extension, and lymph node metastasis, except for those with distant 
Table 3 Cox proportional hazards regression model analysis of overall survival (OS) and disease-specific survival (DSS) of patients stratified by patients' features

\begin{tabular}{|c|c|c|c|c|}
\hline & \multicolumn{2}{|l|}{ OS } & \multicolumn{2}{|l|}{ DSS } \\
\hline & $\operatorname{aHR}(95 \% \mathrm{Cl})$ & $P$-value* & $\operatorname{aHR}(95 \% \mathrm{Cl})$ & $P$-value* \\
\hline \multicolumn{5}{|l|}{ Tumor size $<2 \mathrm{~cm}$} \\
\hline No RAI therapy & Reference & & Reference & \\
\hline RAI therapy & $0.65 \mathrm{I}(0.563,0.752)$ & $<0.001$ & 0.785 (0.47I, I.823) & 0.352 \\
\hline \multicolumn{5}{|l|}{ Tumor size $\geq 2 \mathrm{~cm}$} \\
\hline No RAI therapy & Reference & & Reference & \\
\hline RAI therapy & $0.509(0.430,0.603)$ & $<0.001$ & $0.509(0.430,0.603)$ & $<0.001$ \\
\hline \multicolumn{5}{|l|}{ Age $<45$ years } \\
\hline No RAI therapy & Reference & & Reference & \\
\hline RAI therapy & $0.591(0.418,0.836)$ & 0.003 & $1.846(0.227,14.978)$ & 0.566 \\
\hline \multicolumn{5}{|l|}{ Age $\geq 45$ years } \\
\hline No RAI therapy & Reference & & Reference & \\
\hline RAI therapy & $0.584(0.520,0.657)$ & $<0.001$ & $0.582(0.445,0.760)$ & $<0.001$ \\
\hline \multicolumn{5}{|c|}{ Intrathyroidal extension } \\
\hline No RAI therapy & Reference & & Reference & \\
\hline RAI therapy & $0.638(0.557,0.730)$ & $<0.001$ & $1.042(0.648,1.677)$ & 0.856 \\
\hline \multicolumn{5}{|l|}{ MEE } \\
\hline No RAI therapy & Reference & & Reference & \\
\hline RAI therapy & $0.742(0.493$, I.II I5) & $<0.001$ & $1.356(0.5 \mid 2,3.592)$ & $0.54 I$ \\
\hline \multicolumn{5}{|l|}{ GEE } \\
\hline No RAI therapy & Reference & & Reference & \\
\hline RAI therapy & $0.498(0.395,0.606)$ & $<0.001$ & $0.387(0.278,0.538)$ & $<0.001$ \\
\hline \multicolumn{5}{|l|}{ LNM negative } \\
\hline No RAI therapy & Reference & & Reference & \\
\hline RAI therapy & $0.623(0.545,0.713)$ & $<0.001$ & $0.700(0.462,1.062)$ & 0.094 \\
\hline \multicolumn{5}{|l|}{ LNM positive } \\
\hline No RAI therapy & Reference & & Reference & \\
\hline RAI therapy & $0.539(0.443,0.654)$ & $<0.001$ & $0.498(0.356,0.697)$ & $<0.001$ \\
\hline \multicolumn{5}{|c|}{ No distant metastases } \\
\hline No RAI therapy & Reference & & Reference & \\
\hline RAI therapy & $0.603(0.538,0.676)$ & $<0.001$ & $0.644(0.479,0.865)$ & 0.003 \\
\hline \multicolumn{5}{|l|}{ Distant metastases } \\
\hline No RAI therapy & Reference & & Reference & \\
\hline RAI therapy & $0.418(0.262,0.669)$ & $<0.001$ & $0.378(0.209,0.683)$ & 0.001 \\
\hline
\end{tabular}

Note: *P-values calculated by multivariate Cox analysis.

Abbreviations: aHR, adjusted hazard ratio (adjusted for age at diagnosis, sex, race, region, tumor size, extension, lymph nodes metastases, distant metastases, radiotherapy and surgery); MEE, minimal extrathyroidal extension; GEE, gross extrathyroidal extension; LNM, lymph nodes metastases; RAI, radioactive iodine.

metastasis. Podnos et al suggested that post-surgical RAI therapy could only improve the survival in PTC patients older than 45 years with tumors $>2 \mathrm{~cm}$, involving lymph node and distant metastases. ${ }^{9}$ Some studies stated that surgery alone for small tumors $(<1.5 \mathrm{~cm})$ had favorable prognosis and RAI was not required. In addition, RAI did not influence the recurrence rates in papillary thyroid micro-carcinoma even for those with disease in lymph nodes. ${ }^{18-20}$ Mazzaferri et al demonstrated that RAI therapy significantly reduced recurrence rate and cancerrelated death in patients with tumors $>1.5 \mathrm{~cm} .{ }^{10}$ Other studies revealed that RAI benefited patients with tumors $>1 \mathrm{~cm}$ or residual and metastatic disease after surgery. ${ }^{16,21}$ These results were consistent with ours. For patients with tumors $<2 \mathrm{~cm}$ and without distant metastasis, total or nearly total thyroidectomy had favorable prognosis, and RAI ablation could not improve it, except for those with distant metastasis. Surgery alone could successfully remove the remnant, even in patients with extrathyroidal extension or lymph node metastasis. A recent study demonstrated that minimal extrathyroidal extension did not affect the survival in most well differentiated thyroid cancer, and those patients should have a more aggressive surgical or RAI treatment. ${ }^{22}$

In our study, RAI therapy did not show any benefits in patients with minimal extrathyroidal extension, but multivariate analysis demonstrated that minimal extrathyroidal extension was an independent risk factor for DSS. 


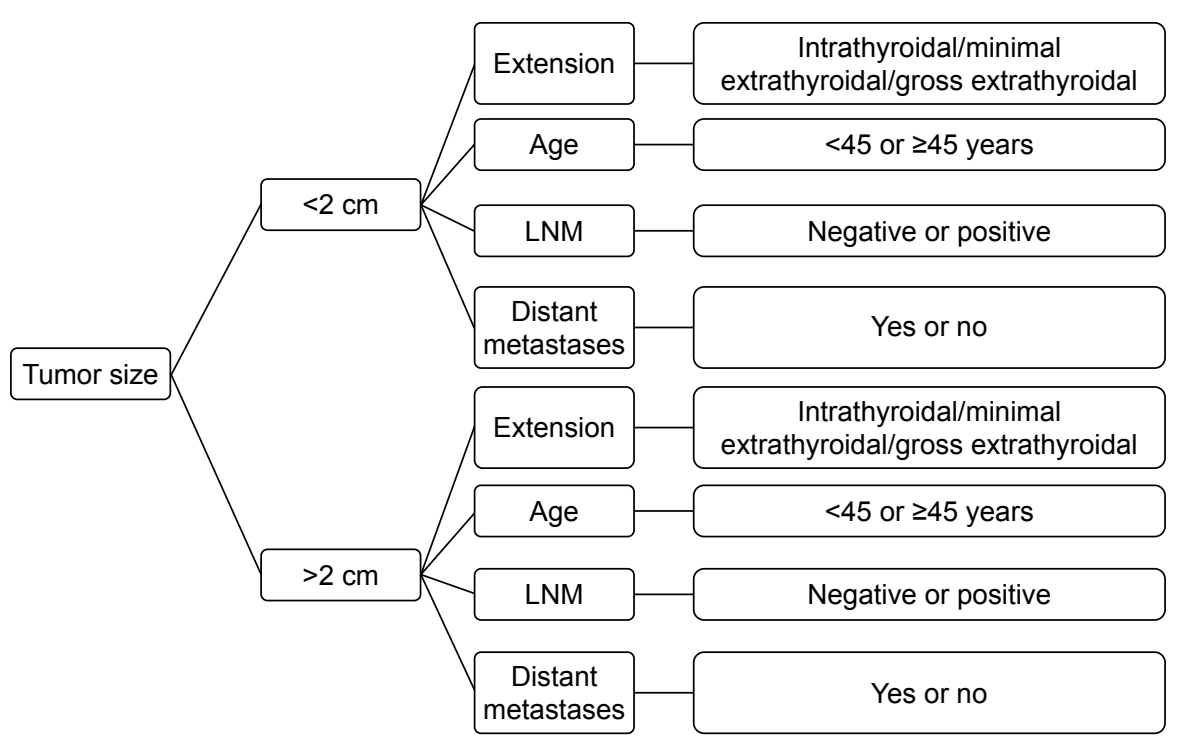

Figure I Stratification of patients based on tumor size, tumor extension, age, lymph node and distant metastases.

Abbreviation: LNM, lymph node metastasis.

For patients with tumors $>2 \mathrm{~cm}$, completeness of surgical resection is hard to achieve, especially when the tumor has invaded into the trachea, larynx or esophagus. Gross extrathyroidal extension has been regarded as a poor prognostic feature for many years. ${ }^{13}$ It is reported that many patients with extrathyroidal extension responded very well to RAI. ${ }^{23}$ However, it remains controversial whether the presence of lymph node metastasis is a risk factor of mortality. ${ }^{24}$ Previous

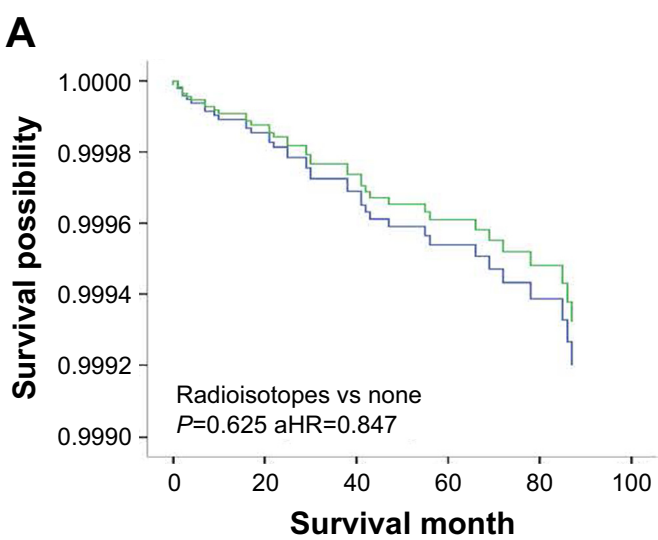

C

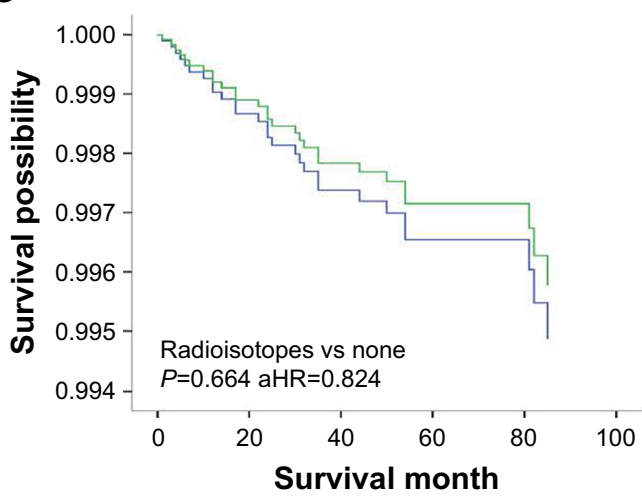

B

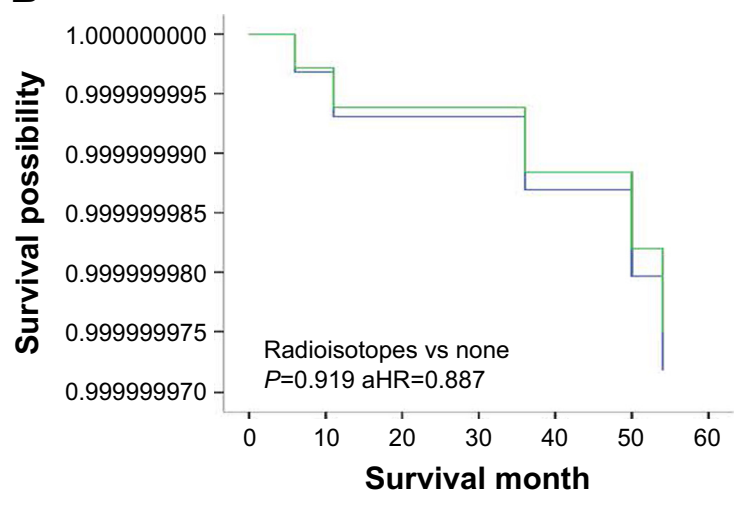

D

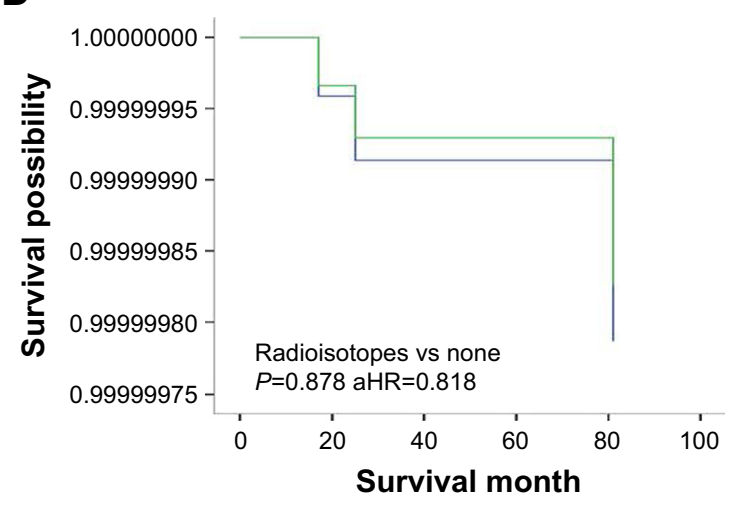

Radiation

$\neg$ None $\rightarrow$ Radioactive iodine

Figure 2 (Continued) 

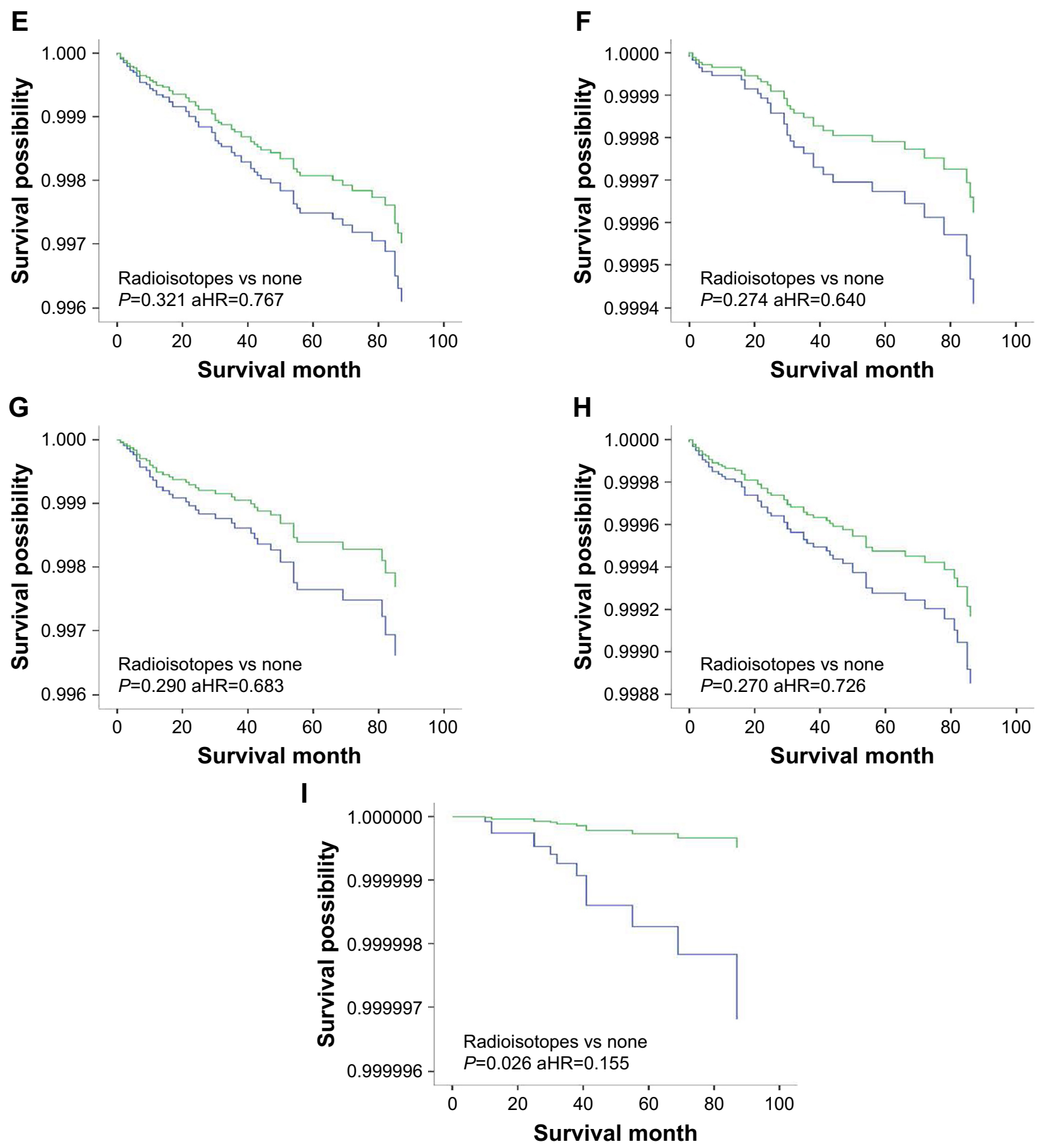

\section{Radiation \\ $\neg$ None $\rightarrow$ Radioactive iodine}

Figure 2 DSS curves of multivariate Cox analysis in the group with tumors $<2 \mathrm{~cm}$. DSS is based on radioactive isotope in (A) patients with intrathyroidal extension; (B) patients with minimal extrathyroidal extension; (C) patients with gross extrathyroidal extension; (D) patients younger than 45 years; (E) patients older than 45 years; (F) patients without lymph node metastases; (G) patients with lymph node metastases; $(\mathbf{H})$ patients without distant metastases; (I) patients with distant metastases. Abbreviations: aHR, adjusted hazard ratios; DSS, disease-specific survival.

studies showed that lymph node metastasis increased the recurrence rate and decreased the survival rate in patients older than 45 years. ${ }^{25-27}$ In our study, patients with lymph node metastasis had poor OS and DSS. Recent ATA guidelines also recommended prophylactic central-compartment neck dissection in patients with PTC clinically involved lateral neck nodes. ${ }^{7}$ RAI ablation eliminated potential tumor nest remnants in the lymph nodes after surgery, thus improving the DSS. Another study also considered lymph node metastasis as an indication of RAI. ${ }^{28}$ Age was another risk factor of recurrence and death. ${ }^{26,29}$ In our study, all patients older than 45 years benefited from RAI therapy. 
Our goals with RAI therapy are to eradicate any normal residual thyroid tissue and locoregional or distant tumor deposits, thereby increasing the sensitivity and specificity of follow-up examinations of persistence or recurrence, such as thyroglobulin measurement and the whole-body radioiodine scan. However, treatment with ${ }^{131}$ I may have dose-dependent adverse effects: dysfunction of salivary gland, reduction of tear production, transient dysfunction of male/female gonad, and higher risk of developing other cancers. It is important to consider these adverse effects when choosing RAI therapy in patients with PTC. ${ }^{30}$ The adverse effects of RAI were not further examined in our study due to the lack of records of them in the SEER database.

There are several limitations in our study. We did not stratify patients into low- or high-risk groups using the current risk stratification systems. To determine whether RAI therapy benefited the survival, we classified patients into different groups with specific features, and we found that tumor size, extension, age, lymph node and distant metastases should be considered in RAI therapy. This retrospective

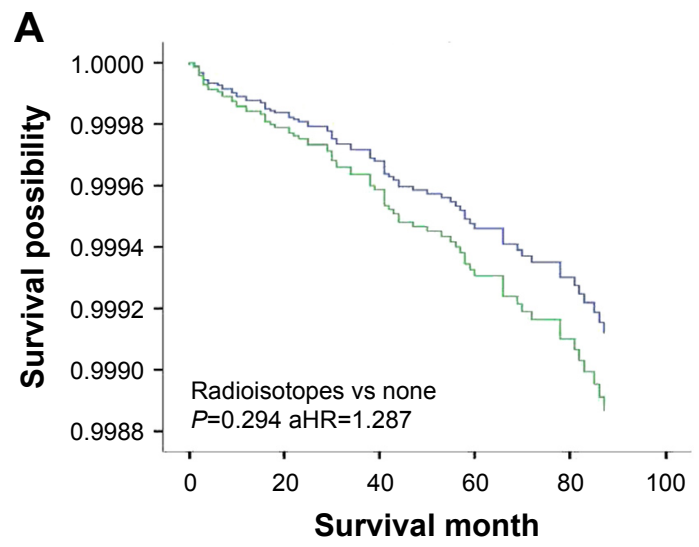

B
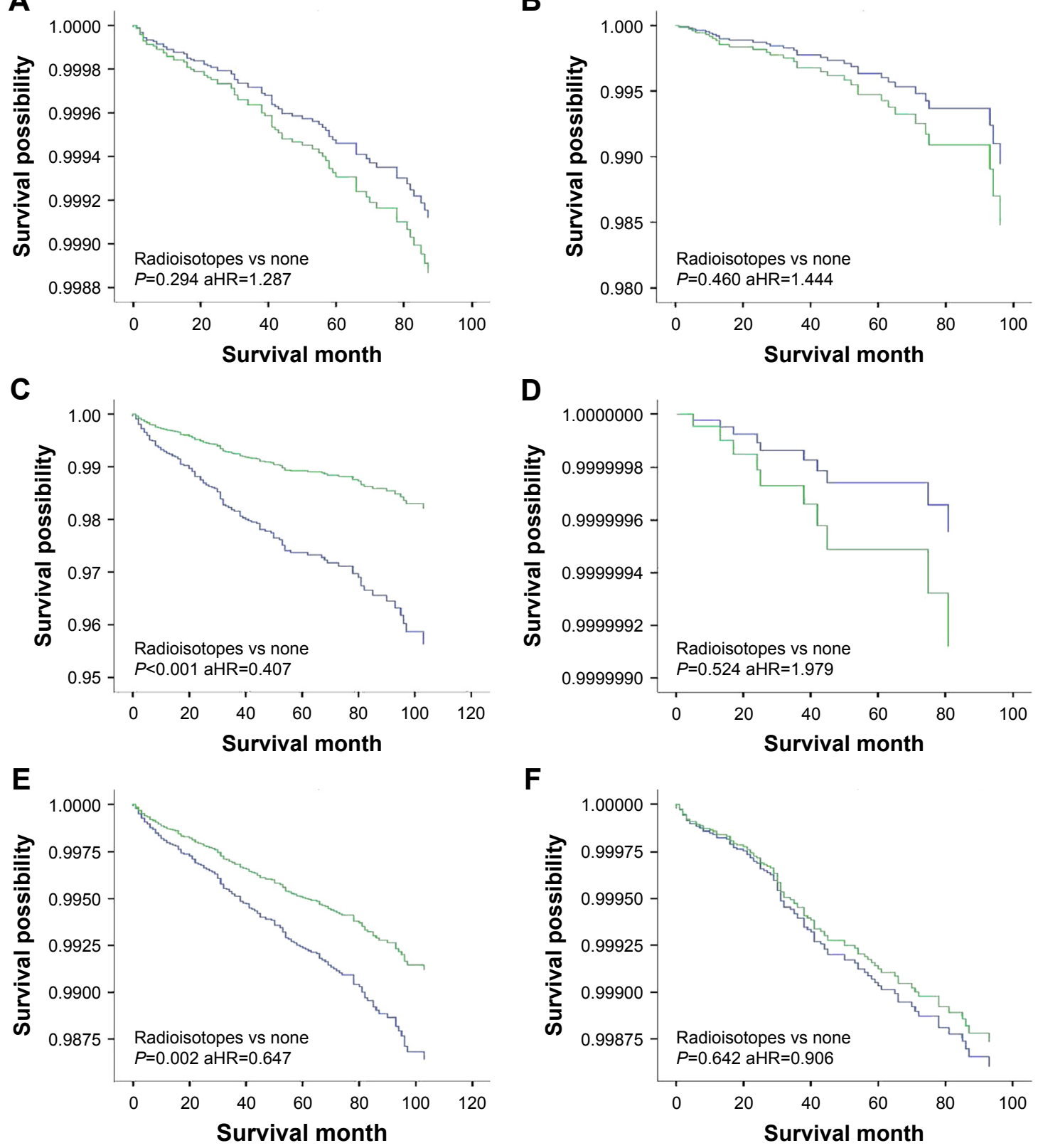

\begin{tabular}{|l|l|}
\hline Radiation \\
$\neg$ None $\neg$ Radioactive iodine \\
\hline
\end{tabular}

Figure 3 (Continued) 

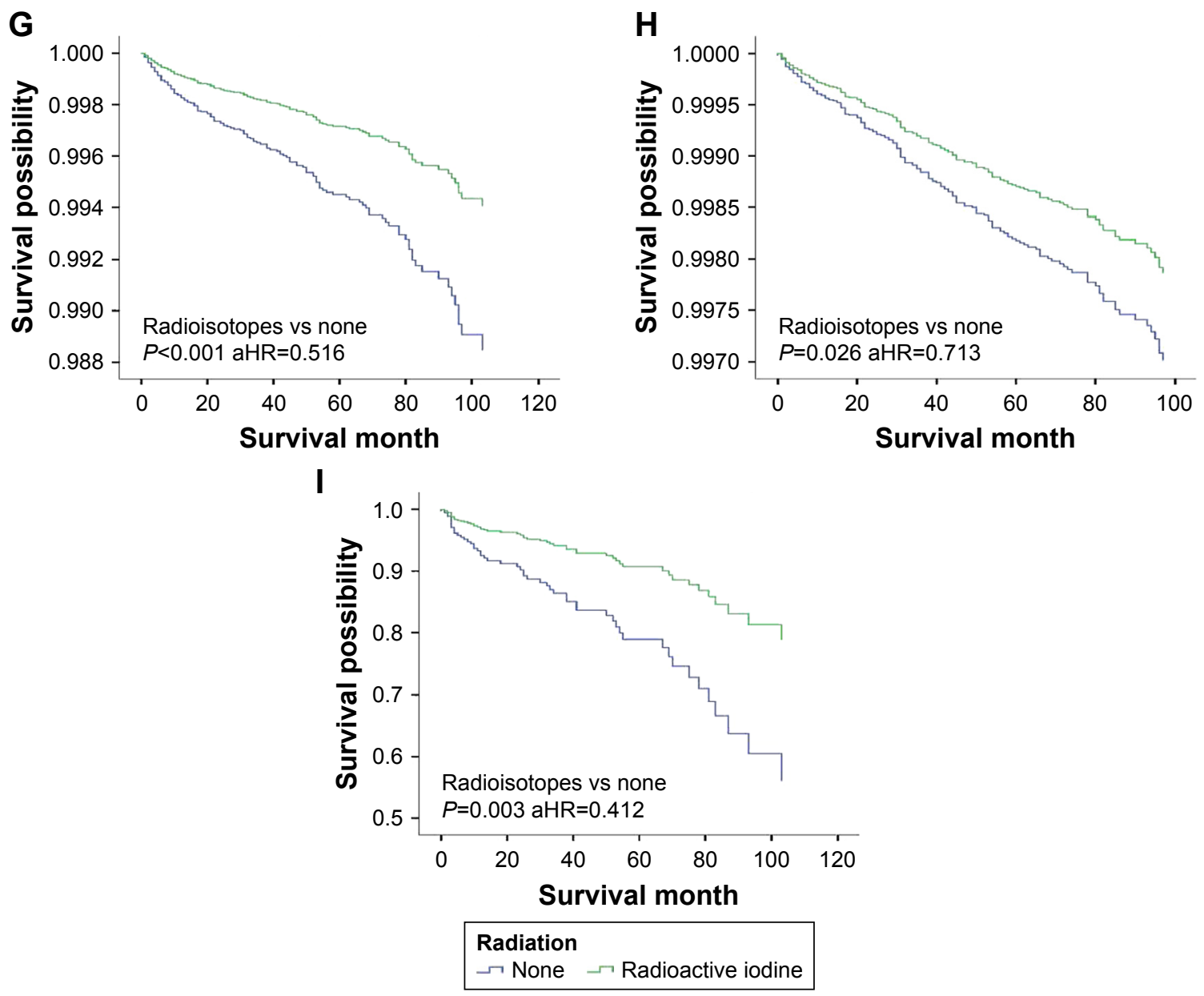

Figure 3 DSS curves of multivariate Cox analysis in the group with tumors $>2 \mathrm{~cm}$. DSS is based on radioactive isotope in (A) patients with intrathyroidal extension; (B) patients with minimal extrathyroidal extension; (C) patients with gross extrathyroidal extension; (D) patients younger than 45 years; (E) patients older than 45 years; (F) patients without lymph node metastases; (G) patients with lymph node metastases; (H) patients without distant metastases; (I) patients with distant metastases. Abbreviations: aHR, adjusted hazard ratios; DSS, disease-specific survival.

analysis was potentially biased by prejudices: those receiving RAI had generally more risk factors (larger tumor size, extrathyroidal extension, lymph nodes and distant metastases) and a more advanced stage of disease; they tend to have a poorer prognosis, thus the survival benefit of RAI therapy would be cancelled by the worse disease features. To decrease the bias caused by patients' characteristics, we categorized patients into different groups (Figure 1), and we found that only those who had relatively high risk factors benefited from RAI therapy. In addition, molecular markers were not included in SEER. It is reported that $B R A F$ had clinical value in planning the treatment strategy. $B R A F$ was associated with more aggressive clinical behaviors, thus it predicted higher necessity of postoperative RAI ablation. ${ }^{31}$ The SEER database has recorded no information about recurrence. Due to the indolent nature of PTC, recurrence is more meaningful than death. Despite these limitations, SEER is still important in understanding the associations between treatment outcomes and specific risk factors. Long-term randomized trials are needed for further study.

\section{Conclusion}

RAI ablation should be recommended to patients with tumors $<2 \mathrm{~cm}$ with distant metastasis or tumors $>2 \mathrm{~cm}$ with one of the following factors: gross extrathyroidal extension, age $>45$ years, lymph node and distant metastases. To optimize the treatment strategy for PTC patients, it is very important to consider all the risk factors.

\section{Acknowledgment}

We thank Qi Wu for assistance in improving the quality of language and revising the statistical methods.

\section{Author contributions}

All authors contributed toward data analysis, drafting and critically revising the paper and agree to be accountable for all aspects of the work. 


\section{Disclosure}

The authors report no conflicts of interest in this work.

\section{References}

1. Lim H, Devesa SS, Sosa JA, Check D, Kitahara CM. Trends in thyroid cancer incidence and mortality in the United States, 1974-2013. JAMA. 2017;317(13):1338-1348.

2. Cady B, Sedgwick CE, Meissner WA, Wool MS, Salzman FA, Werber J. Risk factor analysis in differentiated thyroid cancer. Cancer. 1979; 43(3):810-820.

3. Tala H, Tuttle RM. Contemporary post surgical management of differentiated thyroid carcinoma. Clin Oncol (R Coll Radiol). 2010; 22(6):419-429.

4. Grigsby PW, Reddy RM, Moley JF, Hall BL. Contralateral papillary thyroid cancer at completion thyroidectomy has no impact on recurrence or survival after radioiodine treatment. Surgery. 2006;140(6): 1043-1047; discussion 1047-1049.

5. Ms HHL, FRACS, Kai PW. Postablation stimulated thyroglobulin level is an important predictor of biochemical complete remission after reoperative cervical neck dissection in persistent/recurrent papillary thyroid carcinoma. Ann Surg Oncol. 2013;20(2):653-659.

6. Wartofsky L, Van Nostrand D. Radioiodine treatment of well-differentiated thyroid cancer. Endocrine. 2012;42(3):506-513.

7. Haugen BR, Alexander EK, Bible KC, et al. 2015 American Thyroid Association management guidelines for adult patients with thyroid nodules and differentiated thyroid cancer: the American Thyroid Association Guidelines Task Force on Thyroid Nodules and Differentiated Thyroid Cancer. Thyroid. 2016;26(1):1-133.

8. Luster M, Clarke SE, Dietlein M, et al. Guidelines for radioiodine therapy of differentiated thyroid cancer. Eur J Nucl Med Molecular Imaging. 2008;35(10):1941-1959.

9. Podnos YD, Smith DD, Wagman LD, Ellenhorn JD. Survival in patients with papillary thyroid cancer is not affected by the use of radioactive isotope. J Surg Oncol. 2007;96(1):3-7.

10. Mazzaferri EL, Jhiang SM. Long-term impact of initial surgical and medical therapy on papillary and follicular thyroid cancer. Am J Med. 1994;97(5):418-428.

11. Jonklaas J, Sarlis NJ, Litofsky D, et al. Outcomes of patients with differentiated thyroid carcinoma following initial therapy. Thyroid. 2006;16(12):1229-1242.

12. Grebe SK, Hay ID. Follicular cell-derived thyroid carcinomas. Cancer Treat Res. 1997;89(89):91-140.

13. Tsang RW, Brierley JD, Simpson WJ, Panzarella T, Gospodarowicz MK, Sutcliffe SB. The effects of surgery, radioiodine, and external radiation therapy on the clinical outcome of patients with differentiated thyroid carcinoma. Cancer. 1998;82(2):375-388.

14. Tubiana $M$, Schlumberger M, Rougier $P$, et al. Long-term results and prognostic factors in patients with differentiated thyroid carcinoma. Cancer. 1985;55(4):794-804.

15. Luster M, Reiners C. Radioiodine therapy in differentiated thyroid cancer. World J Endocr Surg. 2009;1(1):7-12.

16. Pacini F, Schlumberger M, Dralle H, et al. European consensus for the management of patients with differentiated thyroid carcinoma of the follicular epithelium. Eur J Endocrinol. 2006;154(6):787-803.

OncoTargets and Therapy

\section{Publish your work in this journal}

OncoTargets and Therapy is an international, peer-reviewed, open access journal focusing on the pathological basis of all cancers, potential targets for therapy and treatment protocols employed to improve the management of cancer patients. The journal also focuses on the impact of management programs and new therapeutic agents and protocols on

Submit your manuscript here: http://www.dovepress.com/oncotargets-and-therapy-journal
17. Silberstein EB, Alavi A, Balon HR, et al. Society of Nuclear Medicine Procedure Guideline for Therapy of Thyroid Disease with Iodine-131 (Sodium Iodide). Society of Nuclear Medicine. Available from: https:// www.researchgate.net/publication/240636680_Society_of_Nuclear_ Medicine_Procedure_Guideline_for_Therapy_of_Thyroid_Disease_ with_Iodine131_Sodium_Iodide. Accessed May 10, 2018.

18. Baudin E, Travagli JP, Ropers J, et al. Microcarcinoma of the thyroid gland: the Gustave-Roussy Institute experience. Cancer. 1998; 83(3):553-559.

19. Hay ID, Grant CS, van Heerden JA, Goellner JR, Ebersold JR, Bergstralh EJ. Papillary thyroid microcarcinoma: a study of 535 cases observed in a 50-year period. Surgery. 1992;112(6):1139-1146; discussion 1146-1137.

20. Palimeri S, Gousis P, Vlassopoulou B. Indications of radioactive iodine ablation in papillary thyroid cancer. Hell Cheirourgike. 2015; 87(1):53-57.

21. American Thyroid Association Guidelines Taskforce on Thyroid Nodules and Differentiated Thyroid Cancer, Cooper DS, Doherty GM et al. Revised American Thyroid Association management guidelines for patients with thyroid nodules and differentiated thyroid cancer. Thyroid. 2009;19(11):1167-1214.

22. Al-Qurayshi Z, Shama MA, Randolph GW, Kandil E. Minimal extrathyroidal extension does not affect survival of well-differentiated thyroid cancer. Endocr Relat Cancer. 2017;24(5):221-226.

23. Lee N, Tuttle M. The role of external beam radiotherapy in the treatment of papillary thyroid cancer. Endocr Relat Cancer. 2006;13(4): 971-977.

24. Lee YD. Surgical strategy for papillary thyroid microcarcinoma. J Korean Thyroid Assoc. 2014;7(1):48-56.

25. Schneider DF, Elfenbein D, Lloyd RV, Chen H, Sippel RS. Lymph node metastases do not impact survival in follicular variant papillary thyroid cancer. Ann Surg Oncol. 2015;22(1):158-163.

26. Yu XM, Wan Y, Sippel RS, Chen H. Should all papillary thyroid microcarcinomas be aggressively treated? An analysis of 18,445 cases. Ann Surg. 2011;254(4):653-660.

27. Zaydfudim V, Feurer ID, Griffin MR, Phay JE. The impact of lymph node involvement on survival in patients with papillary and follicular thyroid carcinoma. Surgery. 2008;144(6):1070-1078.

28. Chow SM, Law SC, Mendenhall WM, et al. Papillary thyroid carcinoma: prognostic factors and the role of radioiodine and external radiotherapy. Int J Radiat Oncol Biol Phys. 2002;52(3):784-795.

29. Mazurat A, Torroni A, Hendrickson-Rebizant J, Benning H, Nason RW, Pathak KA. The age factor in survival of a population cohort of welldifferentiated thyroid cancer. Endocr Connect. 2013;2(3):154-160.

30. Clement SC, Peeters RP, Ronckers CM, et al. Intermediate and longterm adverse effects of radioiodine therapy for differentiated thyroid carcinoma - a systematic review. Cancer Treat Rev. 2015;41(10): 925-934.

31. Han SA, Park WS, Jang JH, Min SY, Ryu JK, Song JY. BRAF mutation may predict higher necessity of postoperative radioactive iodine ablation in papillary thyroid cancer. Ann Surg Treat Res. 2014;87(4): 174-179.

32. Fritz AG. International classification of diseases for oncology: ICD-O. World Health Organization; 2000.

patient perspectives such as quality of life, adherence and satisfaction. The manuscript management system is completely online and includes a very quick and fair peer-review system, which is all easy to use. Visit http://www.dovepress.com/testimonials.php to read real quotes from published authors. 